\title{
Epigenetic dysregulation of endometriosis susceptibility genes (Review)
}

\author{
NATSUKI KOIKE, YUMI HIGASHIURA, JURIA AKASAKA, CHIHARU UEKURI, \\ FUMINORI ITO and HIROSHI KOBAYASHI
}

Department of Obstetrics and Gynecology, Nara Medical University, Kashihara, Nara 634-8522, Japan

Received January 4, 2015; Accepted March 5, 2015

DOI: $10.3892 / \mathrm{mmr} .2015 .3635$

\begin{abstract}
The aim of the present review was to illustrate how dysregulation of hormonal signaling regulates expressional changes of spatially associated genes in endometriosis. From a multi-platform endometriosis dataset, an integrated analysis was performed of epigenomic changes of several biologically relevant genes that have been validated in the literature. Estrogen receptor (ER) may act as a direct epigenetic driver for endometriosis establishment, maintenance and progression. A majority of endometriosis susceptibility genes may be present in functional downstream targets of ER and located near the known imprinting genes. Previous studies have shed light on the overlapping genetic signatures between endometriosis development and the defective decidualization process. The steroid hormone-mediated decidualization signaling pathway was shown to be frequently dysregulated in endometriosis. DNA methylation is associated with various intragenic or intergenic epigenetic modifications of chromatin. Chromatin architecture may be established in temporal and spatial orchestration of the recruitment of genes specifically downregulated in endometriosis. In conclusion, defective chromatin architecture at the ER target locus may have a key role in endometriosis. Endometriosis represents an interesting model to explore the variation of expression of spatially associated genes.
\end{abstract}

Correspondence to: Dr Hiroshi Kobayashi, Department of Obstetrics and Gynecology, Nara Medical University, 840 Shijo-cho, Kashihara, Nara 634-8522, Japan

E-mail: hirokoba@naramed-u.ac.jp

Abbreviations: ER, estrogen receptor; $\mathrm{CDKN}$, cyclin-dependent kinase inhibitor; CEBPA, CCAAT/enhancer-binding protein alpha; CTCF, CCCTC-binding factor; HOXA10, homeobox A10; PR, progesterone receptor

Key words: endometriosis, imprinting, epigenetics, chromatin architecture

\section{Contents}

1. Introduction

2. Aberrant expression of candidate and susceptibility genes

3. Estrogen receptor (ER) as an epigenetic driver

4. Defective chromatin organization and remodeling

5. Somatic mutations of, AT rich interactive domain $1 \mathrm{~A}$ (SWI-like) (ARID1A)

6. Defective chromatin interactions of ER with its inducible genes

7. CCCTC-binding factor (CTCF)-mediated chromatin interactions

8. Mechanistic link between genomic imprinting and endo metriosis-associated methylation profiles

9. Conclusions

\section{Introduction}

Endometriosis affects $\sim 10 \%$ of women of reproductive age and is associated with dysmenorrhea, pelvic pain, infertility and in rare cases with malignant transformation. It is an estrogen-dependent, progesterone-resistant disorder, which contributes to endometriotic cell proliferation and survival (1). Estrogen has been shown to have an important role in the growth of endometriotic lesions (1). Epidemiological studies have suggested that gene polymorphism of estrogen and progesterone receptors is associated with a risk of endometriosis $(2,3)$. Furthermore, several studies have been designed to identify candidate genes and their pathways of endometriosis susceptibility $(4,5)$. In line with those of earlier studies, the results showed that the candidate pathways, which included genes with functions as hormonal regulators, cell cycle regulators, signal transducers, transcription factors, hormones, cytokines, chemokines and (pro)inflammatory molecules, proteases, as well as molecules with functions in cell adhesion, motility, stress response, detoxification, immune response and metabolism, may be associated with susceptibility to endometriosis $(4,5)$.

In addition, aberrations of components at the genetic, epigenetic, transcriptional, post-transcriptional, translational and post-translational level may drive candidate pathways that may affect the susceptibility to this disorder. It is now widely accepted that epigenetic mechanisms critically repress, 
maintain or induce encoded genetic information (6). Silencing of endometriosis-susceptibility genes by epigenetic aberrations has been reported (4). Among the candidate genes, the differentially methylated genes are often associated with steroidogenesis, implantation and placental development (6). These results indicated that numerous genes which are downregulated in the eutopic endometrium of endometriosis are decidualization-associated genes.

Alterations in the gene expression profile influence susceptibility to endometriosis; however, the potential mechanism that drives these alterations has remained elusive. The aim of the present review was to understand how aberrant hormonal signaling is integrated to generate a transcriptional output during the process of endometriosis development.

\section{Aberrant expression of candidate and susceptibility genes}

Steroid receptor-mediated tissue-specific complex functions, including DNA methylation and transcriptional repression signaling are the most affected pathways in the eutopic and ectopic endometrium in women with endometriosis (7). Certain studies proposed a molecular model stating that hypomethylation of the ER- $\beta$ promoter results in overexpression of ER- $\beta$ and reduced expression of ER- $\alpha$ and progesterone receptor (PR) in endometriotic stromal cells (8). In conjunction with estrogen-priming, PR signaling is also involved in a variety of reproductive functions, including embryo implantation, trophoblast invasion, decidualization and subsequent formation of a functional placenta that all are crucial for establishment and maintenance of pregnancy. Two major PR isoforms, PR-A and PR-B, have been identified (9). PR-A acts as a repressor of $\mathrm{PR}-\mathrm{B}$, a stronger activator of progesterone target genes. A reduced PR-B/PR-A ratio has been demonstrated in ectopic tissue, as the promoter region of PR-B is specifically hypermethylated (10). Therefore, a number of progesterone target genes that are essential for implantation and pregnancy were dysregulated in eutopic endometrium of women affected with endometriosis (11). Abnormalities of the epigenome, chromatin remodeling in response to steroid hormones and the decidualization process may contribute to progesterone resistance commonly observed in women with this disease.

A previous review analyzed 20 published datasets that are specifically regulated during the process of decidualization (12). Genetic and epigenetic studies identified a plethora of candidate/susceptibility genes and their downstream targets with potential functional roles in decidualization. During the process of decidualization, estrogen and progesterone bind to their endometrial receptors and activate downstream targets, including HOXA10, FOXO1, C/EBP- $\beta$ and IHH, which in turn regulate cell differentiation, resulting in an endometrium receptive to embryo implantation (Fig. 1). Abrogation of these genes causes stromal cell defects due to deregulation of implantation and decidualization (13).

Recent studies based on (epi)genome-wide technology have noted specific expression of candidate genes and susceptibility genes for endometriosis (12). Reduced expression of the decidualization susceptibility genes whose expression is regulated by transcription factors (HOXA10, FOXO1 and
C/EBP- $\beta$ ), growth factors (the IGF and IGFBP families), cell cycle regulators (the CDKN family) and cytokines/chemokines (LIF, IL11 and TGF- $\beta$ ) can influence the expression of specific target genes and proteins with central roles in the development of endometriosis (12). Bioinformatics analysis revealed a substantial degree of overlapping candidate gene transcriptomes between decidualization process and endometriosis from an (epi)genetic point of view (5). Downregulated genes identified in endometriosis among genes overexpressed in the decidualization process encode proteins directly involved in hormonal regulation as well as transcription factors. Interestingly, in endometriosis, $28 \%$ of differentially overexpressed genes are markedly downregulated during the decidualization process (12). This overlap is significantly greater than expected by chance. The expression pattern of the endometriosis susceptibility genes resembles that of the defective decidualization process. These findings support the hypothesis that retrograde menstruation of regurgitated endometrial cells with insufficient decidualization possibly caused by epigenetic aberrations is implicated in the pathogenesis of endometriosis.

\section{Estrogen receptor (ER) as an epigenetic driver}

Epigenetic modifications control cell development, differentiation and programming. Alterations encompassing epigenetic changes have a key role in regulating gene expression through post-translational modifications of histones (histone methyltransferases, histone acetyltransferases and histone deacteylases), DNA methylation, microRNA silencing, non-coding RNA, long intergenic non-coding RNA and DNA repair mechanisms (14). Recent analyses identified several genes that were hypoacetylated in endometriosis. These include ER- $\alpha$, homeobox A10 (HOXA10), CCAAT/enhancer-binding protein alpha (CEBPA), p16 (INK4a, CDKN2A), p21 (Waf1/Cip1, CDKN1A), p27 (Kip1, CDKN1B), checkpoint kinase 2 (CHEK2), death receptor 6 (DR6), and E-cadherin (CDH1) (15). An inverse correlation between the histone acetylation status and DNA methylation in regulating gene silencing is well established.

Analysis of cytosine-phosphate-guanine $(\mathrm{CpG})$ dinucleotides identified $>40,000$ CpGs that were differentially methylated in endometriosis (16). Promoter hypermethylation may contribute to the understanding of epigenetic regulation in this disorder. DNA hypermethylation in endometriosis affects the expression of several key genes, including PR-B, HOXA10, insulin-like growth factor binding protein 1 (IGFBP1), steroidogenic factor 1 (NR5A1) and aromatase (CYP19A1) (16). Highly focused deviations in endometriotic cell DNA methylation may affect key genes involved in progesterone responsiveness and the decidualization process. As shown in Fig. 1, hypermethylation of the PR-B-specific promoter is considered to be a cause for the development of progesterone resistance. ER activation may be a main driving force for the progression to endometriosis. Expression levels of ER- $\alpha$ in endometriosis were shown to be lower than those in the eutopic endometrium, while ER- $\beta$ was overexpressed in endometriotic stromal cells as compared with that in endometrial cells (17). An increased ER- $\beta$ /ER- $\alpha$ ratio in endometriosis compared to that in endometrial tissues is associated with 


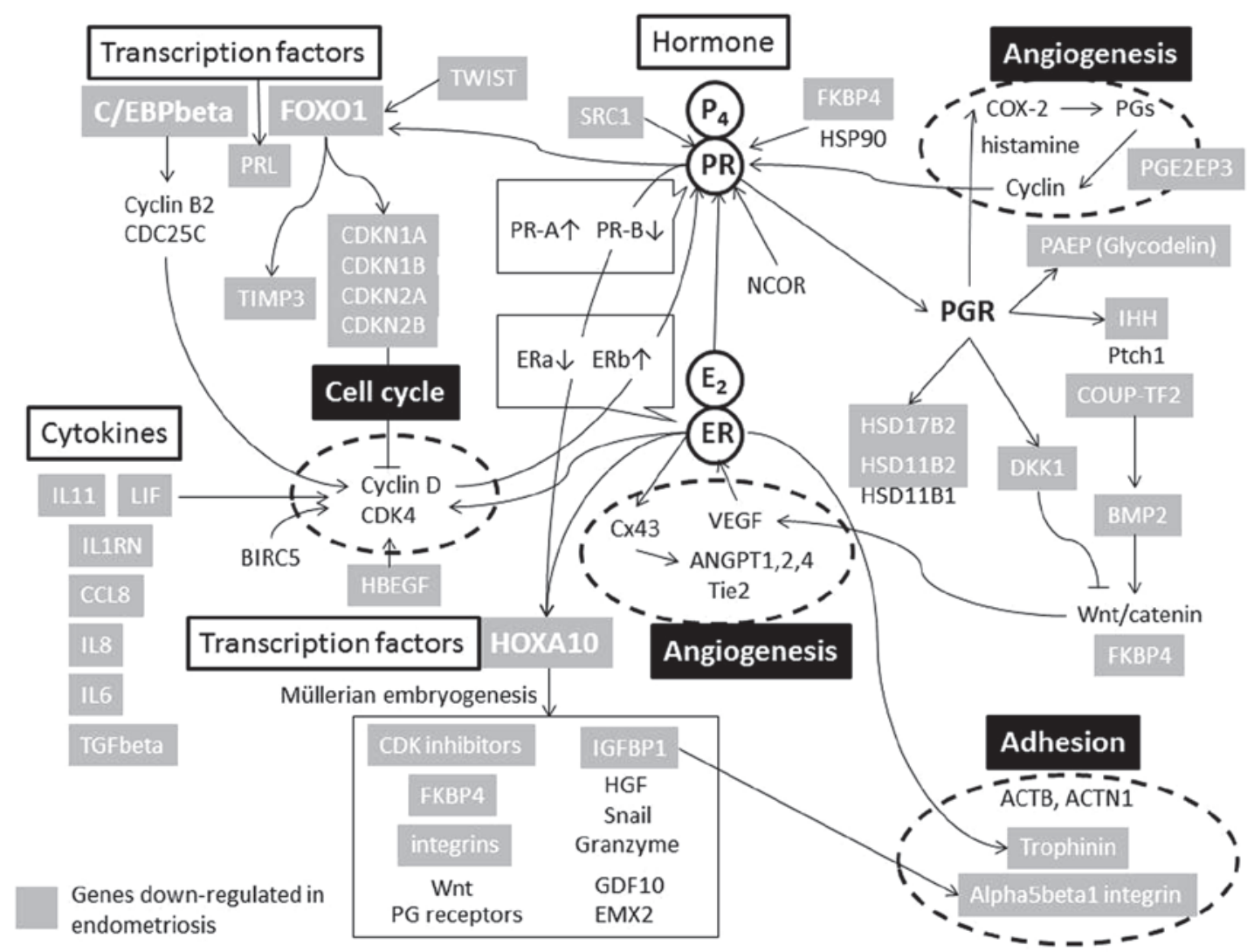

Figure 1. ER and PR-dependent signaling pathways that positively regulate the decidualization process. Compared with non-decidualization endometrial tissues, numerous genes were up-regulated in decidualization tissues, which can fully account for the phenotypic differences. Given the critical role that ER and PR-dependent signaling pathways have in establishing and maintaining the decidualization process, it is particularly important to establish the targets downstream of this transcriptional network. The ER/PR-target genes and the downstream pathway are depicted. A majority of genes was reported to be downregulated in endometriosis, which is often associated with phenotypic changes. This figure was adopted from ref. 12, Fig. 1, and modified in detail.

\section{Chromosome number}

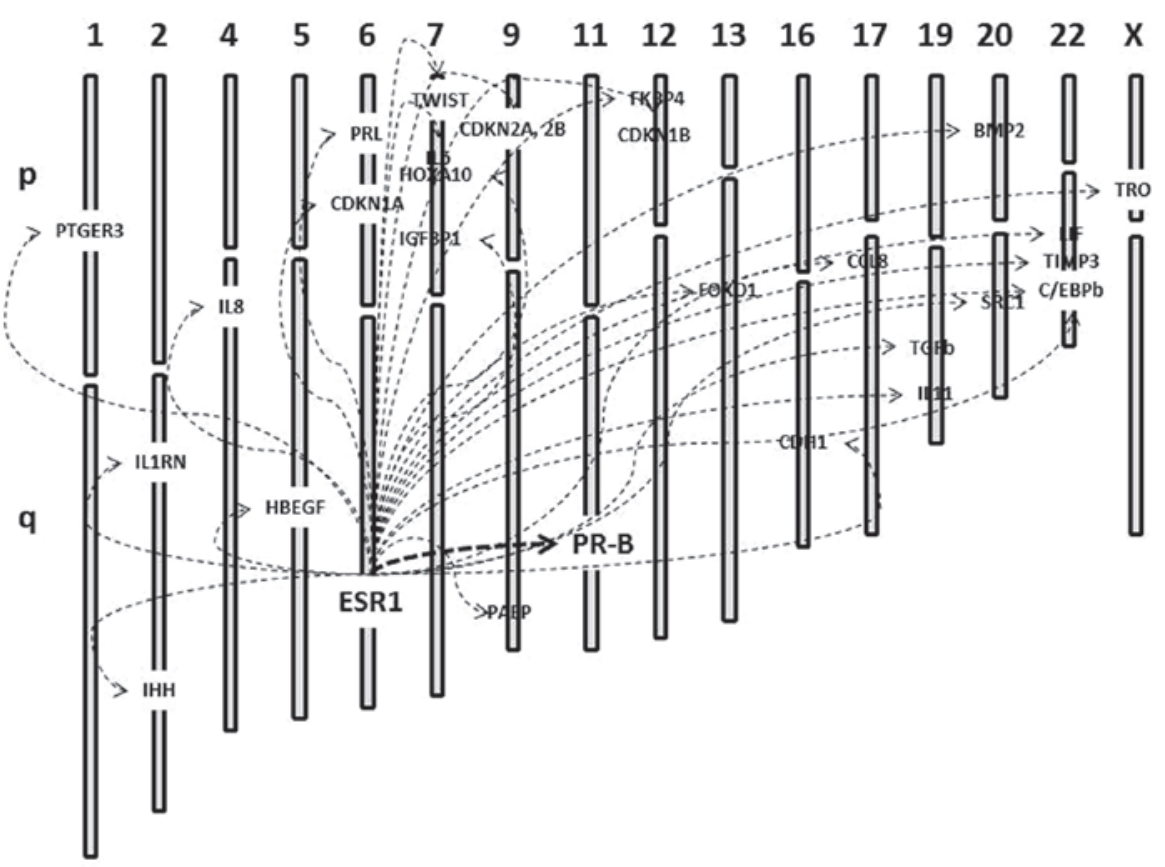

Figure 2. Geographical interactions between ER gene and endometriosis susceptibility genes. Specific gene transcription can be regulated by different chromatin remodeling and various chromatin architectures, including histone displacement, DNA looping, histone variants, histone modification and chromosome territories (29). Long-range topological interactions between different chromosomes may occur preferentially between genomic regions containing the same chromatin marks. This figure was adopted from ref. 4, Fig. 1, and modified in detail. 
Table I. Genes downregulated in endometriosis $(n=29)$.

\begin{tabular}{ll}
\hline Function & \\
\hline Cytokines/chemokines & \\
Cell cycle & LIF, IL6, IL8, IL11, TGFB1, IL1RN, CCL8, BMP2 \\
Transcription factors & CDKN1A, CDKN1B, CDKN2A, CDKN2B \\
Hormones & FOXO1, HOXA10, CEBPB, TWIST1 \\
Growth factors & FKBP4, PRL, PGR \\
Signaling & IGFBP1, HBEGF, SRC \\
Adhesion & IHH, DKK1, PTGER3 \\
Immune system & CDH1, TRO \\
Proteases & PAEP \\
\hline
\end{tabular}

suppressed PR levels, contributing to the loss of progesterone signaling or progesterone resistance (7). Total PR and PR-B were lower in endometriotic stromal cells compared to those in endometrial cells. Several lines of evidence suggested that endometriosis is a chronic disease with substantial epigenetic dysregulation, leading to the imbalance between estrogen and progesterone $(2,3)$.

For the present review, nine published datasets of endometriosis susceptibility genes were analyzed (4,10,18-24). Table I summarizes 29 genes silenced by epigenetic aberration. A majority of endometriosis-specific downregulated genes overlap with those known to be regulated by ER or its downstream targets. These data revealed important ER-dependent signaling pathways, regulatory association and molecular connectivity among the downregulated genes identified in the eutopic endometrium of women affected with endometriosis (12). ER may act as a direct epigenetic driver for endometriosis. Therefore, the ER-dependent (epi)genomic regulation provides novel insight into the pathogenesis of endometriosis.

\section{Defective chromatin organization and remodeling}

Recent studies may support defective chromatin remodeling in endometriosis. DNA methylation and histone modification are interrelated in regulating chromatin remodeling and gene expression (25-28). Knowledge of the role of three-dimensional (3D) architecture of chromatin on the transcriptional regulation is evolving.

\section{Somatic mutations of AT-rich interactive domain $1 \mathrm{~A}$ (SWI-like) (ARID1A)}

Whole-exome sequencing identified that genes involved in chromatin-remodeling complexes are frequently altered in endometriosis, suggesting that genetic alterations in chromatin-remodeling proteins may be involved in the pathogenesis of this disorder (25). Somatic mutations of a chromatin remodeler, such as ARID1A, were frequently found in the endometriotic cyst epithelium in direct continuity with the carcinoma (26). It has been reported that defective or remodeled chromatin organization has a key role in tumor development and growth (27). One may speculate that chromatin architectures present in normal endometrial cells may be lost in endometriotic cells.

\section{Defective chromatin interactions of ER with its inducible genes}

ER binding to chromatin promotes correct assembly of the transcriptional machinery of its target genes (28). As shown in Fig. 2 (29), interactions of ER with its inducible target genes located in the same or different chromosomes may be regulated by $3 \mathrm{D}$ long-distance chromatin interactions $(27,28)$. Future studies will provide a catalog of aberrations pertinent to chromatin topology that contribute to endometriosis development. The 3D organization of the genome may achieve proper temporal and spatial patterns of gene expression. ER is distributed within (near the promoters of estrogen-regulated genes) and outside (distal regions) of gene bodies (27). ER has numerous binding sites along the genome and forms robust long-range enhancer-promoter interactions as an intrachromosomal looping. In addition, these remote binding sites are able to communicate with their putative target genes via long-distance interchromosomal interactions (30). We hypothesize that a majority of endometriosis susceptibility genes may be present in downstream targets of ER and associated with not only various intragenic or intergenic chromatin epigenetic features, but also long-range topological interactions among different chromosomes. Modified chromatin interactions may be created in temporal orchestration of the recruitment of transcription factors specifically downregulated in endometriosis (Table I).

\section{CCCTC-binding factor (CTCF)-mediated chromatin interactions}

CTCF, a zinc finger DNA binding protein, is one of the most important transcription factors that participate in numerous processes associated with global chromatin organization and remodeling (31). CTCF is the vertebrate insulator protein that affects gene expression by mediating intra- and interchromosomal interactions (32). This transcription factor functions mainly as a transcriptional repressor, including hormone-responsive gene silencing, long-range chromatin interactions, enhancer blocking and/or barrier gene insulation, genomic imprinting, and X-chromosome inactivation (33). CTCF can regulate a wide range of target gene functions over long distances in the genome by promoting the formation of chromatin loops. DNA loops form between ER bound 
to enhancer regions and promoter regions of target genes, suggesting the possible importance of CTCF in the 3D chromatin reorganization of the ER-specific target gene loci (34). It has been reported that ER activates its downstream target HOXA10 (16,35). CTCF functions as a controller of HOXA cluster silencing (36). CTCF also acts upstream of the FOX gene and influences differential interactions between ER and chromatin (37). CTCF binding to the specific insulator sites of the target gene loci with a tight loop formation contributes to its silencing. Loss of the CTCF machinery may result in defective chromatin architecture at the ER target locus. We hypothesized that the CTCF-mediated chromatin conformational 3D architecture may be dysregulated in endometriosis. It remains largely unexplored, however, whether chromatin loops are lost during gene repression in endometriosis.

\section{Mechanistic link between genomic imprinting and endometriosis-associated methylation profiles}

Several studies have identified the entirely different DNA methylation profiles in endometriosis, which include a large number of genes associated with transcription factors (HOX and GATA) and steroidogenesis (NR5A1, STAR, STRA6 and HSD17B2) $(6,16)$. However, it remains elusive whether tissue-specific global alterations in the methylome profile pattern occur in endometriosis. The extent to which histone/DNA methylation contributes to its pathogenesis also remains elusive.

To date, two studies investigating this context have been published $(4,38)$. Firstly, the presence of hypermethylated regions located at the ends of chromosomes among endometriosis samples suggested that the distribution pattern of the hypermethylation was not random (38). This indicated that altered non-random methylation of $\mathrm{CpG}$ sites which do not carry the mutation has a possible role in the development of endometriosis. Secondly, among the 29 hypermethylated genes in endometriosis, 19 genes $(65.5 \%)$ were located near the known imprinted foci (4). Genes designated to genomic imprinting are associated with allele-specific expression. Imprinted genes are highly conserved and organized in clusters. According to an imprinting gene database (http://www. geneimprint.com/site/home), >100 imprinted genes have been identified in humans thus far (39). It was demonstrated that a number of hypermethylated genes were located in close proximity to the imprinted foci, which was significantly higher than expected by chance.

\section{Conclusions}

Several lines of evidence have indicated that endometriosis is an epigenetic disease. Firstly, bioinformatics analysis revealed a substantial degree of overlapping transcriptomes between endometriosis candidate genes and the decidualization process (5). Interestingly, in endometriosis, 28\% differentially overexpressed genes are markedly downregulated during the decidualization process in endometrial stromal cells (12). Downregulated genes identified in endometriosis among genes overexpressed in the decidualization process encode proteins directly involved in cytokine/chemokine signaling, cell cycle regulation, adhesion, the immune system, as well as transcription factors, hormones, growth factors and proteases (12) (Table I). In addition to the genes described in Fig. 2, several important genes, including DNA methyltransferases (40), COX-2 (41), estrogen receptor 2 (ESR2) (42) and steroidogenic factor-1 (SF-1) (43) are aberrantly methylated. The mechanism responsible for insufficient decidualization may be associated with the development of endometriosis. Consistent with this hypothesis, enhanced estrogenic activity and reduced progesterone responsiveness are considered to be involved in the etiology of endometriosis (11). Therefore, endometriosis is a chronic disease with substantial epigenetic dysregulation of decidualization susceptibility genes (4).

Secondly, a large number of endometriosis susceptibility genes are frequently associated with downstream targets of ER. Approximately two thirds of the downregulated genes associated with endometriosis susceptibility are not random, but instead are located near the known paternally or maternally expressed imprinting genes (4). Hypermethylated regions showed a pattern of non-random distribution, which serves to facilitate coordinated transcriptional modulation in groups of genes (38). There appears to be a functional association in the genomic location between genes specifically downregulated in endometriosis and epigenetically imprinted genes.

Finally, various chromatin architectures can reportedly regulate ER-mediated gene expression, supporting a role for long-range topological interactions in ER biology (28). CTCF was shown to have a role in ER-mediated gene expression (28). The transcription factor CTCF is involved in imprinting, long-range chromatin interactions and transcription. Loss of CTCF machinery or chromatin remodeling may lead to defective chromatin architecture at the ER target locus. However, a global analysis of the role of CTCF in chromatin looping, chromatin remodeling, various chromatin architectures and the physical access of distal chromosomes in endometriotic stromal cells has yet to be performed. Further studies are required for molecular (epi)genomic approaches based on the chromosome conformation capture method in the ER target gene regions, and to then identify elements that can act over a distance.

In conclusion, transcriptional repression at the ER target locus may be an emerging key factor in endometriosis, possibly through the non-random distribution of various DNA epigenetic architectures, including histone acetylation, methylation, CTCF-dependent insulation, genomic imprinting and 3D chromatin features. Additional studies are required to further elucidate the association of transcriptional profiles with defective chromatin architectures in endometriosis.

\section{Acknowledgements}

The present review was supported by a grant-in-aid for the Scientific Research from the Ministry of Education, Science, and Culture of Japan to H.K (grant no. 26293361).

\section{References}

1. Tamaresis JS, Irwin JC, Goldfien GA, Rabban JT, Burney RO, Nezhat C, DePaolo LV and Giudice LC: Molecular classification of endometriosis and disease stage using high-dimensional genomic data. Endocrinology 155: 4986-4999, 2014. 
2. Hsieh YY, Wang YK, Chang CC and Lin CS: Estrogen receptor alpha-351 XbaI*G and -397 PvuII'C-related genotypes and alleles are associated with higher susceptibilities of endometriosis and leiomyoma. Mol Hum Reprod 13: 117-122, 2007.

3. Wieser F, Schneeberger C, Tong D, Tempfer C, Huber JC and Wenzl R: PROGINS receptor gene polymorphism is associated with endometriosis. Fertil Steril 77: 309-312, 2002.

4. Kobayashi H, Higashiura Y, Koike N, Akasaka J, Uekuri C, Iwai K, Niiro E, Morioka S and Yamada Y: Genes downregulated in endometriosis are located near the known imprinting genes. Reprod Sci 21: 966-972, 2014.

5. Kobayashi H, Uekuri C and Shigetomi H: Towards an understanding of the molecular mechanism of endometriosis: Unbalancing epithelial-stromal genetic conflict. Gynecol Endocrinol 30: 7-15, 2014.

6. Yamagata Y, Nishino K, Takaki E, Sato S, Maekawa R, Nakai A and Sugino N: Genome-wide DNA methylation profiling in cultured eutopic and ectopic endometrial stromal cells. PLoS One 9: e83612, 2014.

7. Bulun SE, Monsavais D, Pavone ME, Dyson M, Xue Q, Attar E, Tokunaga $\mathrm{H}$ and $\mathrm{Su}$ EJ: Role of estrogen receptor- $\beta$ in endometriosis. Semin Reprod Med 30: 39-45, 2012.

8. Bulun SE, Cheng YH, Pavone ME, Xue Q, Attar E, Trukhacheva E, Tokunaga H, Utsunomiya H, Yin P, Luo X, et al Estrogen receptor-beta, estrogen receptor-alpha, and progesterone resistance in endometriosis. Semin Reprod Med 28: 36-43, 2010

9. Conneely OM, Maxwell BL, Toft DO, Schrader WT and O'Malley BW: The A and B forms of the chicken progesterone receptor arise by alternate initiation of translation of a unique mRNA. Biochem Biophys Res Commun 149: 493-501, 1987.

10. Wu Y, Strawn E, Basir Z, Halverson G and Guo SW: Promoter hypermethylation of progesterone receptor isoform B (PR-B) in endometriosis. Epigenetics 1: 106-111, 2006.

11. Burney RO, Talbi S, Hamilton AE, Vo KC, Nyegaard M, Nezhat CR, Lessey BA and Giudice LC: Gene expression analysis of endometrium reveals progesterone resistance and candidate susceptibility genes in women with endometriosis. Endocrinology 148: 3814-3826, 2007.

12. Kobayashi H, Iwai K, Niiro E, Morioka S and Yamada Y: Fetal programming theory: Implication for the understanding of endometriosis. Hum Immunol 75: 208-217, 2014.

13. Lim H, Ma L, Ma WG, Maas RL and Dey SK: Hoxa-10 regulates uterine stromal cell responsiveness to progesterone during implantation and decidualization in the mouse. Mol Endocrinol 13: 1005-1017, 1999.

14. Sharma S, Kelly TK and Jones PA: Epigenetics in cancer. Carcinogenesis 31: 27-36, 2010.

15. Nasu K, Kawano Y, Kai K, Aoyagi Y, Abe W, Okamoto M and Narahara H: Aberrant histone modification in endometriosis. Front Biosci (Landmark Ed) 19: 1202-1214, 2014.

16. Dyson MT, Roqueiro D, Monsivais D, Ercan CM, Pavone ME, Brooks DC, Kakinuma T, Ono M, Jafari N, Dai Y, et al: Genome-wide DNA methylation analysis predicts an epigenetic switch for GATA factor expression in endometriosis. PLoS Genet 10: e1004158, 2014.

17. Shao R, Cao S, Wang X, Feng Y and Billig H: The elusive and controversial roles of estrogen and progesterone receptors in human endometriosis. Am J Transl Res 6: 104-113, 2014.

18. Guo SW: Epigenetics of endometriosis. Mol Hum Reprod 15: 587-607, 2009.

19. Monteiro JB, Colón-Díaz M, García M, Gutierrez S, Colón M, Seto E, Laboy J and Flores I: Endometriosis is characterized by a distinct pattern of histone 3 and histone 4 lysine modifications. Reprod Sci 21: 305-318, 2014.

20. Nasu K, Kawano Y, Tsukamoto Y, Takano M, Takai N, Li H, Furukawa Y, Abe W, Moriyama M and Narahara H: Aberrant DNA methylation status of endometriosis: Epigenetics as the pathogenesis, biomarker and therapeutic target. J Obstet Gynaecol Res 37: 683-695, 2011.

21. Izawa M, Taniguchi F, Terakawa N and Harada T: Epigenetic aberration of gene expression in endometriosis. Front Biosci (Elite Ed) 5: 900-910, 2013.

22. Colón-Díaz M, Báez-Vega P, García M, Ruiz A, Monteiro JB, Fourquet J, Bayona M, Alvarez-Garriga C, Achille A, Seto E, et al: HDAC1 and HDAC2 are differentially expressed in endometriosis. Reprod Sci 19: 483-492, 2012.

23. Cakmak H and Taylor HS: Implantation failure: Molecular mechanisms and clinical treatment. Hum Reprod Update 17: 242-253, 2011
24. Kawano Y, Nasu K, Li H, Tsuno A, Abe W, Takai N and Narahara H: Application of the histone deacetylase inhibitors for the treatment of endometriosis: Histone modifications as pathogenesis and novel therapeutic target. Hum Reprod 26 : 2486-2498, 2011.

25. Li X, Zhang Y, Zhao L, Wang L, Wu Z, Mei Q, Nie J, Li X, Li Y, Fu X, et al: Whole-exome sequencing of endometriosis identifies frequent alterations in genes involved in cell adhesion and chromatin-remodeling complexes. Hum Mol Genet 23 6008-6021, 2014

26. Wiegand KC, Shah SP, Al-Agha OM, Zhao Y, Tse K, Zeng T, Senz J, McConechy MK, Anglesio MS, Kalloger SE, et al: ARID1A mutations in endometriosis-associated ovarian carcinomas. N Engl J Med 363: 1532-1543, 2010.

27. Liu MH and Cheung E: Estrogen receptor-mediated long-range chromatin interactions and transcription in breast cancer. Mol Cell Endocrinol 382: 624-632, 2014.

28. Ross-Innes CS, Brown GD and Carroll JS: A co-ordinated interaction between CTCF and ER in breast cancer cells. BMC Genomics 12: 593, 2011

29. Liang XH, Deng WB, Li M, Zhao ZA, Wang TS, Feng XH, Cao YJ, Duan EK and Yang ZM: Egr1 protein acts downstream of estrogen-leukemia inhibitory factor (LIF)-STAT3 pathway and plays a role during implantation through targeting Wnt4. J Biol Chem 289: 23534-23545, 2014.

30. Hu Q, Kwon YS, Nunez E, Cardamone MD, Hutt KR, Ohgi KA, Garcia-Bassets I, Rose DW, Glass CK, Rosenfeld MG, et al: Enhancing nuclear receptor-induced transcription requires nuclear motor and LSD1-dependent gene networking in interchromatin granules. Proc Natl Acad Sci USA 105: 19199-19204, 2008.

31. Franco MM, Prickett AR and Oakey RJ: The role of CCCTC-binding factor (CTCF) in genomic imprinting, development, and reproduction. Biol Reprod 91: 125, 2014.

32. Yang $\mathbf{J}$ and Corces VG: Chromatin insulators: A role in nuclear organization and gene expression. Adv Cancer Res 110: 43-76, 2011.

33. Ong CT and Corces VG. Enhancer function: new insights into the regulation of tissue-specific gene expression. Nat Rev Genet 12: 283-293, 2011.

34. Fullwood MJ, Liu MH, Pan YF, Liu J, Xu H, Mohamed YB, Orlov YL, Velkov S, Ho A, Mei PH, et al: An oestrogen-receptor-alpha-bound human chromatin interactome. Nature 462: 58-64, 2009.

35. Fambrini M, Sorbi F, Bussani C, Cioni R, Sisti G and Andersson KL: Hypermethylation of HOXA10 gene in mid-luteal endometrium from women with ovarian endometriomas. Acta Obstet Gynecol Scand 92: 1331-1334, 2013.

36. Heger P, Marin B, Bartkuhn M, Schierenberg E and Wiehe T: The chromatin insulator CTCF and the emergence of metazoan diversity. Proc Natl Acad Sci USA 109: 17507-17512, 2012.

37. Zhang Y, Liang J, Li Y, Xuan C, Wang F, Wang D, Shi L, Zhang D and Shang Y: CCCTC-binding factor acts upstream of FOXA1 and demarcates the genomic response to estrogen. J Biol Chem 285: 28604-28613, 2010.

38. Borghese B, Barbaux S, Mondon F, Santulli P, Pierre G, Vinci G, Chapron $C$ and Vaiman D: Research resource: Genome-wide profiling of methylated promoters in endometriosis reveals a subtelomeric location of hypermethylation. Mol Endocrinol 24: 1872-1885, 2010

39. Edwards CA and Ferguson-Smith AC: Mechanisms regulating imprinted genes in clusters. Curr Opin Cell Biol 19: 281-289, 2007.

40. Wu Y, Strawn E, Basir Z, Halverson G and Guo SW: Aberrant expression of deoxyribonucleic acid methyltransferases DNMT1 DNMT3A, and DNMT3B in women with endometriosis. Fertil Steril 87: 24-32, 2007

41. Wang D, Chen Q, Zhang C, Ren F and Li T: DNA hypomethylation of the COX-2 gene promoter is associated with up-regulation of its mRNA expression in eutopic endometrium of endometriosis. Eur J Med Res 17: 12, 2012

42. Xue Q, Lin Z, Cheng YH, Huang CC, Marsh E, Yin P, Milad MP, Confino E, Reierstad S, Innes J, et al: Promoter methylation regulates estrogen receptor 2 in human endometrium and endometriosis. Biol Reprod 77: 681-687, 2007.

43. Xue Q, Lin Z, Yin P, Milad MP, Cheng YH, Confino E, Reierstad S and Bulun SE: Transcriptional activation of steroidogenic factor-1 by hypomethylation of the $5^{\prime} \mathrm{CpG}$ island in endometriosis. J Clin Endocrinol Metab 92: 3261-3267, 2007. 